\title{
About the relation between the quasiparticle Green's function in cuprates obtained from ARPES data and the magnetic susceptibility
}

\author{
D. S. Inosov ${ }^{\text {a }}$ S. V. Borisenko ${ }^{\text {a }}$ I. Eremin ${ }^{\text {b }}$ A. A. Kordyuk ${ }^{\text {a,c }}$ V. B. Zabolotnyy ${ }^{a}$ J. Geck $^{a}$ \\ A. Koitzsch ${ }^{\text {a }}$ J. Fink $^{\text {a }}$ M. Knupfer ${ }^{\mathrm{a}}$ B. Büchner ${ }^{\mathrm{a}}$ \\ a Institute for Solid State Research, IFW Dresden, P.O.Box 270116, D-01171 Dresden, Germany \\ ${ }^{\mathrm{b}}$ Max Planck Institute for the Physics of Complex Systems, D-01187 Dresden, Germany \\ ${ }^{\mathrm{c}}$ Institute of Metal Physics of National Academy of Sciences of Ukraine, 03142 Kyiv, Ukraine
}

\begin{abstract}
Angle resolved photoemission spectroscopy (ARPES) provides a detailed view of the renormalized band structure in cuprates and, consequently, is a key to the self-energy and the quasiparticle Green's function. Such information gives a clue to the comparison of ARPES with scanning tunneling microscopy, inelastic neutron scattering (INS), and Raman scattering data. Here we touch on a potential possibility of such a comparison with the dynamical magnetic susceptibility measured in INS experiments. Calculations based on the experimentally measured quasiparticle selfenergies in cuprates lead to the estimated magnetic susceptibility response with many-body effects taken into account.
\end{abstract}

Key words:

cuprate superconductors, dynamical magnetic susceptibility, ARPES, inelastic neutron scattering, itinerant magnetism PACS: 74.72.-h, 74.72.Hs, 74.25.Ha, 74.20.-z, 79.60.-i

The normal-state Lindhard response function (polarization operator) is related to the quasiparticle Green's function via a simple autocorrelation formula [1]:

$\chi_{0}(\mathbf{Q}, \Omega) \propto-2 i \int G(\mathbf{k}, \omega) G(\mathbf{k}+\mathbf{Q}, \omega+\Omega) d^{2} k d \omega(1)$

Apart from the bare band structure, equation (1) also holds for the renormalized Green's function.

Knowing the Lindhard response function $\chi_{0}$ (which is also known as the bare spin susceptibility), one can obtain from RPA the dynamic spin susceptibility $\chi[2]$, the imaginary part of which is directly proportional to the measured INS intensity [3]:

$\chi(\mathbf{Q}, \Omega)=\chi_{0}(\mathbf{Q}, \Omega) /\left[1+J_{Q} \chi_{0}(\mathbf{Q}, \Omega)\right]$

The coefficient $J_{Q}$ in the denominator of (2) describes the effective four-point vertex (Hubbard interaction or superexchange), which can be refined to the required degree of accuracy, although for many applications its $k$-dependence is neglected [4]. In our calculations we assume $J_{Q}=J_{0}\left[\cos Q_{x} a+\cos Q_{y} a\right]$.
Following these formulae, it is straightforward to conclude that knowing the real and imaginary parts of the Green's function leads us to a comparison of ARPES results with the INS data. Although this idea was discussed earlier $[1,4,5,6]$, the calculations based on real ARPES data were not yet performed. The imaginary part of the Green's function is directly related to the measured ARPES intensity (although it can be affected by several factors). The real part can be obtained if one knows the self-energy, which is also a routine self-consistent Kramers-Kronig procedure in the state of the art ARPES data processing $[7,8]$. Thus, it is possible to calculate the magnetic susceptibility response basing on the experimental ARPES spectra.

In the superconducting state, the anomalous Green's function additionally contributes to $\chi_{0}$, which can not be taken into account from ARPES data. On the other hand, as this additional term is additive, we argue that all features observed in the first ("normal") term, including the resonance 

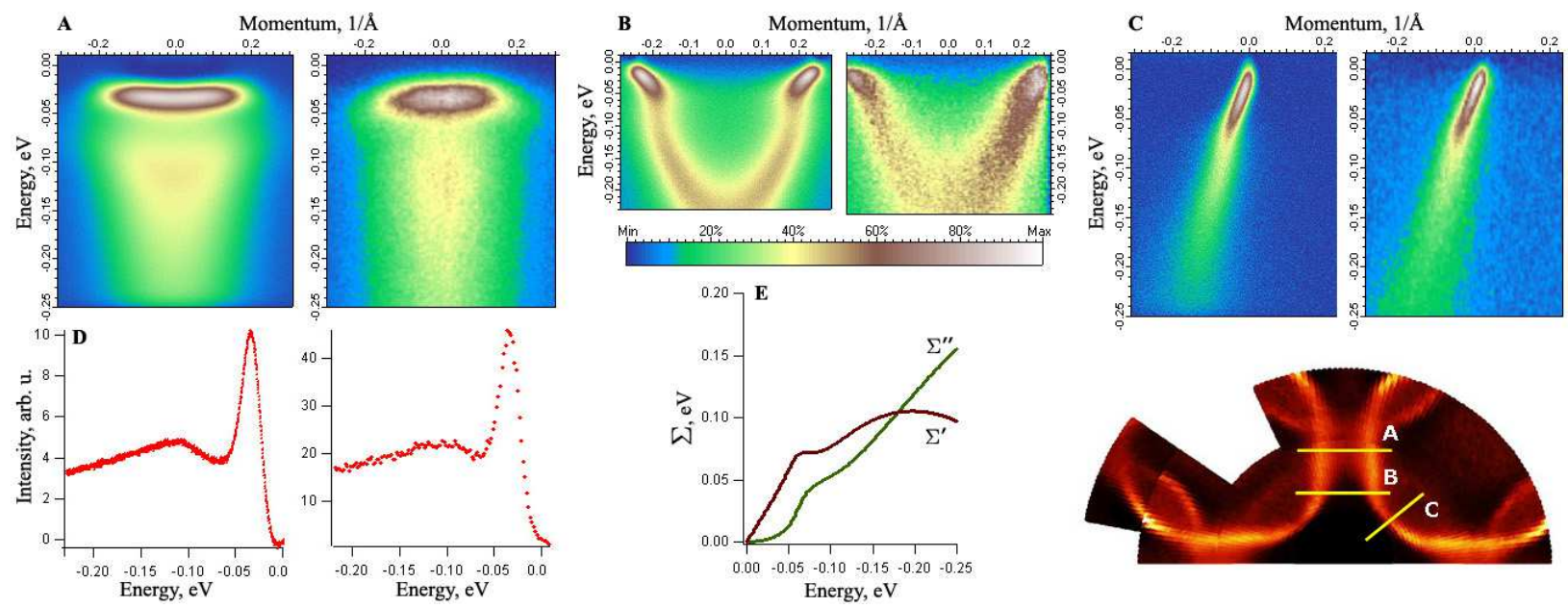

Fig. 1. The modeled (left) and experimental (right) ARPES spectra of the antibonding band at $(\pi, 0)$ point (A), along a shifted cut in the same direction (B) and along the nodal direction (C) in OD Bi-2212 at $50 \mathrm{~K}$. The model images correspond to the $20 \mathrm{meV}$ energy resolution and $0.025 \AA^{-1}$ angular resolution. Panel D shows the energy distribution curves along the dashed lines in the antinodal spectra. The Kramers-Kronig consistent real $\left(\Sigma^{\prime}\right)$ and imaginary $\left(\Sigma^{\prime \prime}\right)$ parts of the nodal self-energy used in the calculation are shown on panel E.

mode, will be still present in the resulting Lindhard function, so we content ourselves with the consideration of the first term only, even in the superconducting state.

In order to exclude the effect of matrix elements and experimental resolution, we modeled the spectra using the self-energy based on the bare electron dispersion studied in [8] and a self-energy model involving quadratic scattering rate, kink of given width, height and position, and a density of states pile-up peak located at the kink energy and characterized by the relative amplitude and width. The real part of the self-energy was calculated by the Kramers-Kronig procedure. Self-energy parameters were specified independently for the nodal and antinodal parts of the spectra, with a d-wave interpolation between these two directions. The superconducting gap was specified in the anti-nodal direction, vanishing in the d-wave manner to the nodes. For posterior calculations, all the free parameters were adjusted during comparison with a set of experimental ARPES spectra in order to achieve the best correspondence (Fig. 1).

Basing on the model dataset built for optimally doped Bi-2212 at $50 \mathrm{~K}$, with the superconducting gap of $30 \mathrm{meV}$, including bonding and antibonding bands with equal intensities, we have calculated the Lindhard function in the energy range down to $0.15 \mathrm{eV}$ below the Fermi level in the whole Brillouin zone. After that we calculated the dynamical spin susceptibility by adjusting the $J_{0}$ parameter trying to reproduce the resonance at $(\pi, \pi)$ and the $45^{\circ}$ ro- tation of the incommensurate peaks that was most clearly observed in the INS experiments on YBCO [9]. The resulting $\chi$ (Fig. 2) qualitatively reproduces the energy resonance at $\sim 40 \mathrm{meV}$ in the $(\pi, \pi)$ point, as well as the four peaks dispersing in the $(0, \pi)$ and $(\pi, 0)$ directions below the resonance and along the Brillouin zone diagonals for higher energies.

The project is part of the Forschergruppe FOR538.
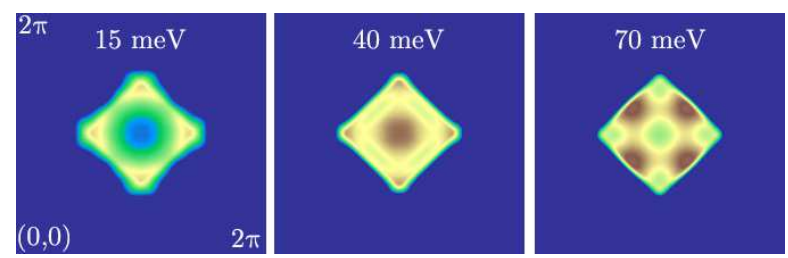

Fig. 2. Constant energy cuts of the dynamical spin susceptibility obtained from the renormalized Lindhard function within the RPA approach. The center of each Brillouin zone image corresponds to the $(\pi, \pi)$ point.

\section{References}

[1] Ar. Abanov et al., Phys. Rev. Lett. 83, 1652 (1999)

[2] D. Z. Liu et al., Phys. Rev. Lett. 75, 4130 (1995)

[3] A. Schnyder et al., cond-mat/0510790 (2005)

[4] M. R. Norman, Phys. Rev. B 61, 14751 (2000)

[5] D. Manske et al., Phys. Rev. B 63, 054517 (2001)

[6] M. Norman, C. Pépin, Rep. Prog. Phys. 66, 1547 (2003)

[7] A. A. Kordyuk et al., Phys. Rev. B 67, 064504 (2003)

[8] A. A. Kordyuk et al., Phys. Rev. B 71, 214513 (2005)

[9] S. M. Hayden et al., Nature 429, 531 (2004) 